\title{
Performance Evaluation of Two Multistatic Radar Detectors on Real and Simulated Sea-Clutter Data
}

\author{
Riccardo Palamà ${ }^{1}$, Luke Rosenberg ${ }^{2}$ and Hugh Griffiths ${ }^{1}$ \\ ${ }^{1}$ University College London, UK \\ ${ }^{2}$ Defence Science and Technology Group, Australia \\ Email: r.palama@ucl.ac.uk
}

\begin{abstract}
In this paper we evaluate the performance of two multistatic radar detection schemes on real and syntheticallygenerated sea clutter data. The analysis utilizes both monostatic and bistatic radar data, simultaneously collected by the nodes of the NetRAD system. The first contribution is a demonstration of how the evolved Doppler spectrum model can accurately simulate multistatic sea-clutter with statistical and spectral properties which match the real data. The second contribution is an analysis of the detection performance obtained from different bistatic angles and a demonstration that the results from both real and simulated data are comparable.
\end{abstract}

Keywords-multistatic radar; detection; sea-clutter

\section{INTRODUCTION}

The goal of a multistatic radar system is to utilize multiple transmitters and receivers to maximize the information extracted from a radar target scene. The geometric diversity provides additional degrees of freedom which can be used to improve the performance of target detection, classification and tracking. This property of multistatic radar systems has often been defined as clutter diversity [1]. In this paper, we consider the improvements achieved in target detection by combining signals from different nodes in a multistatic radar system. Multistatic target detection techniques are usually divided into either centralized or decentralized detectors. For centralized techniques, the central processor receives statistics from each radar node, then computes a global statistic to be compared with the detection threshold. For decentralized techniques, each node performs a threshold comparison, then transmits the resulting decision to the central node, which makes the final decision.

For this work, we consider two centralized multistatic detectors. The first one is a multistatic version of the Normalized Matched Filter (NMF) [2] -[4], which is a suboptimum detector in the case that the disturbance (clutter plus thermal noise) is compound-Gaussian. The second multistatic detector selects the maximum single-channel statistic over all the radar nodes [4]. Both of these techniques are expected to improve the performance when compared to a single-channel equivalent.

To test these detection schemes, we use both real and synthetic sea-clutter data. The real data was collected by the
NetRAD system, which is a multistatic netted radar prototype employed for several measurement campaigns investigating sea-surface scattering [4], detection and tracking of small drones [5] and classification of human micro-Doppler signatures [6].

Accurate simulation of sea-clutter enables stimulation of radar processors during development and testing and to evaluate radar detection algorithms. A simulated signal must faithfully reproduce the characteristics of real clutter including its amplitude statistics, short-term temporal correlation (typically represented by its Doppler spectra) and spatial and longer-term temporal variations. It must also represent the variation of range and azimuth over time as observed from a surveillance radar. The evolving Doppler spectrum model was designed to meet these requirements and has been extensively validated by the CSIR [7], NetRAD [8] and Ingara [9] sea clutter datasets. The key contribution here is to demonstrate how this model can be used to accurately simulate multistatic sea clutter data and verify that the performance of the two multistatic detection schemes matches that achieved using real data.

This paper is organized as follows: Section II introduces the theoretical background of the multistatic detection schemes, Section III describes the system and dataset, Section IV illustrates the modelling and simulation of multistatic sea-clutter data and Section $\mathrm{V}$ examines the performance of the multistatic detectors. Final remarks are then included in Section VI.

\section{THEORETICAL BACKGROUND}

We assume that the multistatic radar system consists of $Q$ transmit-receive nodes or channels with the received signal from the $q$-th node - under the hypotheses $H_{1}$ (target present) and $H_{0}$ (target absent) given by

$$
\begin{cases}H_{1}: & \boldsymbol{z}_{q}=\alpha_{q} \boldsymbol{p}_{q}+\boldsymbol{w}_{q} \\ H_{0}: & \boldsymbol{z}_{q}=\boldsymbol{w}_{q}\end{cases}
$$

where $\mathbf{z}_{\mathrm{q}}$ is the vector collecting $L$ time samples received from the range cell under test within one coherent processing interval (CPI) and $\mathbf{w}_{\mathrm{q}}$ is the interference (clutter plus thermal noise). 
The target signal consists of its complex amplitude, $\alpha_{\mathrm{q}}$, and steering vector, $\boldsymbol{p}_{q}=\left\{\exp \left(-j 2 \pi f_{q} l\right)\right\}_{l=0}^{L-1}$, where $f_{\mathrm{q}}$ is the normalized Doppler frequency of the target, obtained by the projection of the target velocity onto the target-transmitter and target-receiver line-of-sight [3].

If we consider that the statistical distribution of the clutter is a compound-Gaussian, then one common sub-optimal coherent detection technique is the NMF, given by

$$
\varphi_{q}\left(\boldsymbol{p}_{q}, \boldsymbol{z}_{q}, \boldsymbol{R}_{q}\right)=\frac{\left|\boldsymbol{p}_{q}^{H} \boldsymbol{R}_{q}^{-1} \boldsymbol{z}_{q}\right|^{2}}{\left(\boldsymbol{p}_{q}^{H} \boldsymbol{R}_{q}^{-1} \boldsymbol{p}_{q}\right)\left(\mathbf{z}_{q}^{H} \boldsymbol{R}_{q}^{-1} \mathbf{z}_{q}\right)}<^{\lambda}
$$

where the superscript $\mathrm{H}$ indicates a Hermitian transpose, $\mathbf{R}_{\mathrm{q}}$ is the clutter covariance matrix and $\lambda$ is the threshold. In a practical system, the covariance matrix must be estimated from secondary data (i.e. range cells adjacent to the cell under test) and the NMF is then referred to as the Normalized Adaptive Matched Filter (NAMF). If the clutter is stationary, then a larger number of secondary range cells, $K$, yields a more accurate estimate of the covariance. However, sea clutter varies in both range and time and it is often preferable to keep $K$ smaller than the spatial decorrelation length. Similarly, the CPI length should be smaller than the clutter temporal decorrelation time.

In this work, the clutter covariance matrix was estimated using the normalized sample covariance matrix estimate [10] which has been shown to achieve a better accuracy with respect to alternative methods [3]. It is given by

$$
\widehat{\boldsymbol{R}}_{q}=\frac{L}{K} \sum_{k=1}^{K} \frac{\mathbf{z}_{q, k} \mathbf{z}_{q, k}^{H}}{\mathbf{z}_{q, k}^{H} \mathbf{z}_{q, k}}
$$

where the subscript $k$ refers to the $k$-th secondary range cell. In the case of multiple channels, the statistics of $\varphi_{q}\left(\boldsymbol{p}_{q}, \boldsymbol{z}_{q}, \widehat{\boldsymbol{R}}_{q}\right)$ for $q=1, \ldots, Q$ can be combined to calculate a global statistic. If the nodes are independent (i.e. their crosscorrelation is zero), then a useful sub-optimal detector known as the Multichannel Normalized Adaptive Matched Filter (MNAMF) can be used [1]-[4]. It is given by

$$
\boldsymbol{\Lambda}_{\mathrm{M}-\mathrm{NAMF}}(\mathbf{z})=\prod_{q=1}^{Q}\left[1-\varphi_{q}\left(\boldsymbol{p}_{q}, \mathbf{z}_{q}, \widehat{\boldsymbol{R}}_{q}\right)\right]^{-1}{ }^{>} \gamma
$$

where $\gamma$ is the threshold. An alternative multistatic detection rule (M-maxrule) [4] selects the maximum single-channel statistic over all the radar channels,

$$
\boldsymbol{\Lambda}_{\text {Max }}(\mathbf{z})=\max _{q=1, \ldots, Q}\left\{\varphi_{q}\left(\boldsymbol{p}_{q}, \mathbf{z}_{q}, \widehat{\boldsymbol{R}}_{q}\right)\right\}_{<}^{>} \chi
$$

for a threshold $\chi$. While this detector was shown to be inferior to the M-NAMF detector in [4], we would like to test that result here by providing a more detailed analysis with real data.

\section{SYSTEM AND DATASET DESCRIPTION}

The NetRAD system is an S-band multistatic radar system, developed jointly by University College London, UK and the University of Cape Town, South Africa. The main parameters of the NetRAD system are summarized in Table I.

The data analyzed here was collected by the NetRAD system during a measurement campaign in October 2010 at Scarborough, Western Cape, South Africa. Both a monostatic and bistatic node were used for the experiments with a baseline of $1827 \mathrm{~m}$. Measurements were made by varying the azimuth angle, baseline and polarization (horizontally transmit and receive $(\mathrm{HH})$ or vertically transmit and receive $(\mathrm{VV})$ ). For each range bin, the number of received pulses was $N_{p}=130000$ and the bistatic angles, $\beta$ were $60^{\circ}$ and $90^{\circ}$ [11][12]. The wind direction was from the North with a speed that increased during the trials from $10.2 \mathrm{~m} / \mathrm{s}$ to $12.3 \mathrm{~m} / \mathrm{s}$, while the wave height varied between $3.3 \mathrm{~m}$ to $4.2 \mathrm{~m}$, corresponding to a Douglas sea state 5. Both nodes were looking cross-wind with the waves travelling towards the receiver of the bistatic node. Fig. 1 shows the collection geometry, where both monostatic and bistatic measurements were collected for each bistatic angle.

TABLE I. NETRAD SYSTEM PARAMETERS.

\begin{tabular}{|l|l|}
\hline Carrier frequency & $2.4 \mathrm{GHz}$ \\
\hline Transmitted signal & $\mathrm{Up} /$ down chirp \\
\hline Bandwidth & $45 \mathrm{MHz}$ \\
\hline Pulse repetition frequency & $1 \mathrm{kHz}$ \\
\hline Pulse duration & Variable \\
\hline Range resolution & $3.33 \mathrm{~m}$ \\
\hline Sampling frequency & $100 \mathrm{MHz}$ \\
\hline Polarization & $\mathrm{HH}, \mathrm{VV}$ (separately) \\
\hline Half power beamwidth & $9^{\circ}(\mathrm{el}.) \times 11^{\circ}($ az. $)$ \\
\hline
\end{tabular}

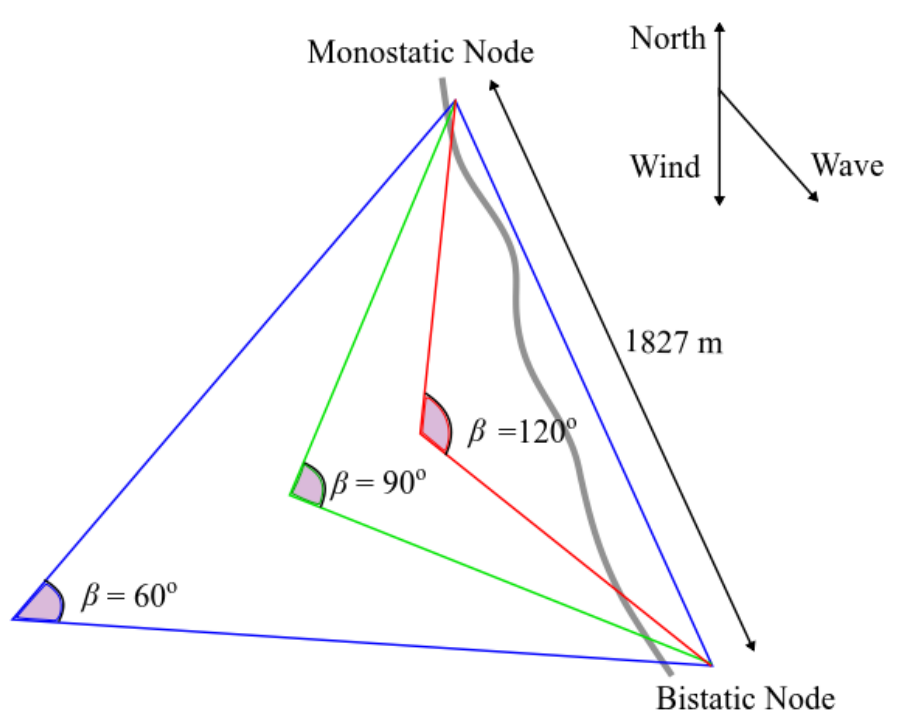

Fig. 1 - Bistatic collection geometry for the NetRAD trials. 


\section{SEA-ClutTER MODELLING AND SimUlation}

Simulating realistic sea clutter requires accurate representation of the amplitude distribution, spatial correlation, the Doppler spectrum, the clutter to noise ratio and the amplitude distribution across frequency. In addition for a multistatic scenario, the cross-correlation of the texture must be modelled.

The evolving spectrum model is able to capture these characteristics and is used for the bistatic simulation. It is based on the compound Gaussian sea clutter model [13]

$$
S(f, x, s)=\frac{x}{\sqrt{2 \pi} s} \exp \left(-\frac{\left(f-m_{\mathrm{f}}(x)\right)^{2}}{2 s^{2}}\right)
$$

where $\mathrm{S}($.$) is the power spectral density for Doppler$ frequency, $f$, and $x$ is the local clutter intensity or texture. $m_{\mathrm{f}}(x)$ is the local mean Doppler frequency given by

$$
m_{\mathrm{f}}(x)=A+B x /\langle x\rangle
$$

and $s$ is the spectrum width (standard deviation of the Gaussian-shaped spectrum), which has a Gaussian probability density function with mean value $m_{s}$ and standard deviation $\sigma_{s}$.

The texture distribution is correlated over time or range with a specified distribution. To achieve a K-distribution for the overall clutter signal, the texture can be modelled with a gamma distribution

$$
P(x)=\frac{b^{v}}{\Gamma(v)} x^{v-1} \exp (-b x)
$$

where $v$ and $b=v / p_{c}$ are the shape and scale parameters respectively with the mean given by $p_{c}$. If we consider a short enough time series, the long term temporal correlation is approximately constant and only the spatial correlation needs to be considered. For this work, we consider a Gaussian model for the spatial correlation

$$
R_{\text {spat }}(r)=\exp \left(-r^{2} / r_{\text {spat }}^{2}\right)
$$

where $r_{\text {spat }}$ is the spatial decorrelation length. For a bistatic simulation, the cross-correlation of the texture, $\rho$, between the two nodes can be simulated by first creating two realizations of the texture, $\tilde{x}_{1}$ and $\tilde{x}_{2}$, each with a desired amplitude distribution and correlation. The cross-correlated texture is then given by

$$
\begin{aligned}
& x_{1}=\tilde{x}_{1}, \\
& x_{2}=\rho \tilde{x}_{1}+(1-\rho) \tilde{x}_{2} .
\end{aligned}
$$

To simulate sea clutter using this model, one method is to first create a realization of the Doppler spectrum over $L$ pulses. The time domain signal is then obtained by an inverse Fourier transform with the final clutter plus noise signal,

$$
Y(f)=\sqrt{S(f, x, s)} C(0,1)+C\left(0, p_{n}\right)
$$

where $p_{n}$ is the noise mean power in the frequency domain and $C(0, p)$ are realizations of complex normal random variables with zero mean and variance $p$.

In previous work [8], many of the evolved Doppler spectrum parameters were estimated from a single snapshot of the data. For this study, the parameters are re-estimated for each different block of data processed in a Monte Carlo detection scheme. An example of these results for each bistatic angle and polarization are summarized in Table II using the methods described in [9].

To demonstrate the model, consider realizations of both the monostatic and bistatic data using a CPI of 64 pulses and 75 range bins. A direct comparison of the Doppler spectra is shown in Figs. 2 and 3 for the $\mathrm{HH}$ and VV polarizations of the $\beta=60^{\circ}$ data set. A good representation of the sea clutter is observed in all cases. The next comparison in Fig. 4 looks at the real and estimated shape variation across frequency for $\beta=60^{\circ}$, where the shape is estimated using the $z \log z$ method [14]. A good match of this parameter is important to make sure that the performance of coherent detectors matches the real data. Again, the simulated results show a good match with the real data.

Table III summarizes the estimated shape, spatial decorrelation length and the root mean square error of the Kdistribution shape across the endo-clutter region of the Doppler spectrum. For each result, there is a reasonable match between the data and simulated results. The largest mismatch is for the $\beta=90^{\circ} \mathrm{VV}$ polarized data with an RMS error of 1.3

TABLE II. NETRAD ESTIMATED PARAMETERS FOR THE EVOLVING DOPPLER SPECTRUM MODEL.

\begin{tabular}{|l|l|l|l|l|}
\hline $\begin{array}{l}\text { Monostatic } \\
\text { Polarization / bistatic angle, } \beta\end{array}$ & $\begin{array}{l}\mathrm{HH}, \\
60^{\circ}\end{array}$ & $\begin{array}{l}\mathrm{VV}, \\
60^{\circ}\end{array}$ & $\begin{array}{l}\mathrm{HH}, \\
90^{\circ}\end{array}$ & $\begin{array}{l}\mathrm{VV}, \\
90^{\circ}\end{array}$ \\
\hline CNR $(\mathrm{dB})$ & 31.2 & 34.0 & 26.5 & 34.1 \\
\hline K-dist. shape, $v$ & 0.3 & 0.9 & 0.6 & 2.0 \\
\hline Spatial decorr. length, $r_{\text {spat }}(\mathrm{m})$ & 0.8 & 1.5 & 1.4 & 0.8 \\
\hline Spectrum mean, A & 6.0 & 12.7 & 14.4 & 11.9 \\
\hline Spectrum slope, B & 0.03 & -0.3 & -0.05 & 0.3 \\
\hline Spectrum width mean, $\mu_{s}$ & 12.7 & 12.4 & 12.7 & 12.1 \\
\hline Spectrum width std., $\sigma_{s}$ & 1.9 & 1.5 & 2.3 & 1.6 \\
\hline Bistatic & \multicolumn{5}{|l}{} \\
\hline CNR $(d B)$ & 20.5 & 27.6 & 26.4 & 30.2 \\
\hline K-dist. shape, $v$ & 0.4 & 1.3 & 0.7 & 1.4 \\
\hline Spatial decorr. length, $r_{\text {spat }}(\mathrm{m})$ & 6.3 & 6.5 & 4.9 & 1.8 \\
\hline Spectrum mean, A & 2.2 & 9.2 & 1.9 & 13.5 \\
\hline Spectrum slope, B & 0.2 & 0.3 & 0.08 & -0.08 \\
\hline Spectrum width mean, $\mu_{s}$ & 12.6 & 12.2 & 11.5 & 12.5 \\
\hline Spectrum width std., $\sigma_{s}$ & 1.9 & 2.1 & 1.7 & 1.9 \\
\hline Texture cross-correlation, $\rho(\%)$ & 6.3 & 2.7 & 7.3 & 6.1 \\
\hline
\end{tabular}

TABLE III. MEASURED PARAMETERS FOR THE SIMULATED DATA.

\begin{tabular}{|l|l|l|l|l|}
\hline $\begin{array}{l}\text { Monostatic } \\
\text { Polarization / bistatic angle, } \beta\end{array}$ & $\begin{array}{l}\mathrm{HH}, \\
60^{\circ}\end{array}$ & $\begin{array}{l}\mathrm{VV}, \\
60^{\circ}\end{array}$ & $\begin{array}{l}\mathrm{HH}, \\
90^{\circ}\end{array}$ & $\begin{array}{l}\mathrm{VV}, \\
90^{\circ}\end{array}$ \\
\hline K-dist. shape, $v$ & 0.4 & 0.9 & 0.7 & 1.8 \\
\hline Spatial decorr. length, $r_{\text {spat }}(\mathrm{m})$ & 1.9 & 1.6 & 1.5 & 1.3 \\
\hline K-dist. shape (freq.) RMS error & 0.15 & 0.5 & 0.6 & 0.9 \\
\hline Bistatic & 0.6 & 0.6 & 0.5 & 1.8 \\
\hline K-dist. shape, $v$ & 6.2 & 7.3 & 4.3 & 1.5 \\
\hline Spatial decorr. length, $r_{\text {spat }}(\mathrm{m})$ & 0.2 & 0.6 & 0.6 & 1.3 \\
\hline K-dist. shape (freq.) RMS error & 0.6 & 7.4 & 9.0 \\
\hline Texture cross-correlation, $\rho(\%)$ & 3.5 & 7.1 & 7.4 &
\end{tabular}



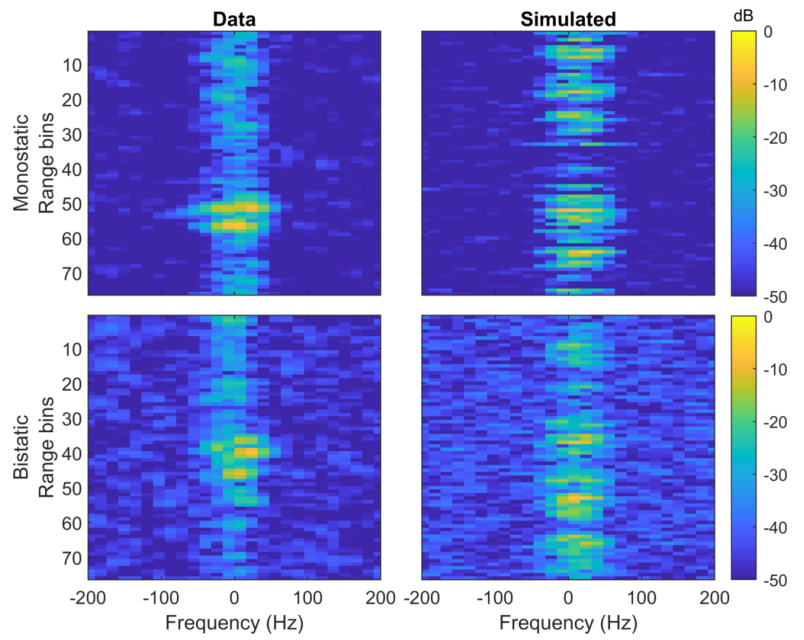

Fig. 2 - Real (left) and simulated (right) Doppler spectrum, HH polarization, $\beta=60^{\circ}$.

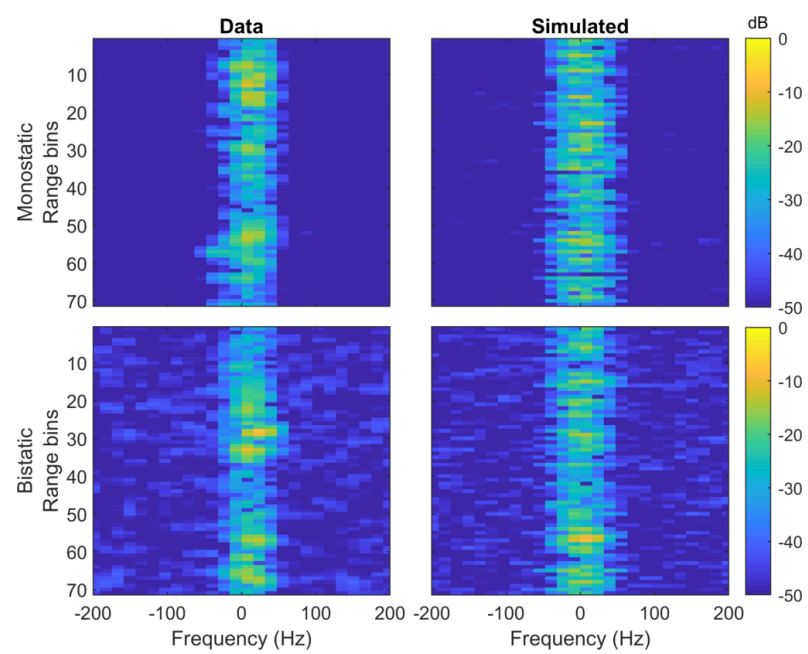

Fig. 3 - Real (left) and simulated (right) Doppler spectrum, VV polarization, $\beta=60^{\circ}$.
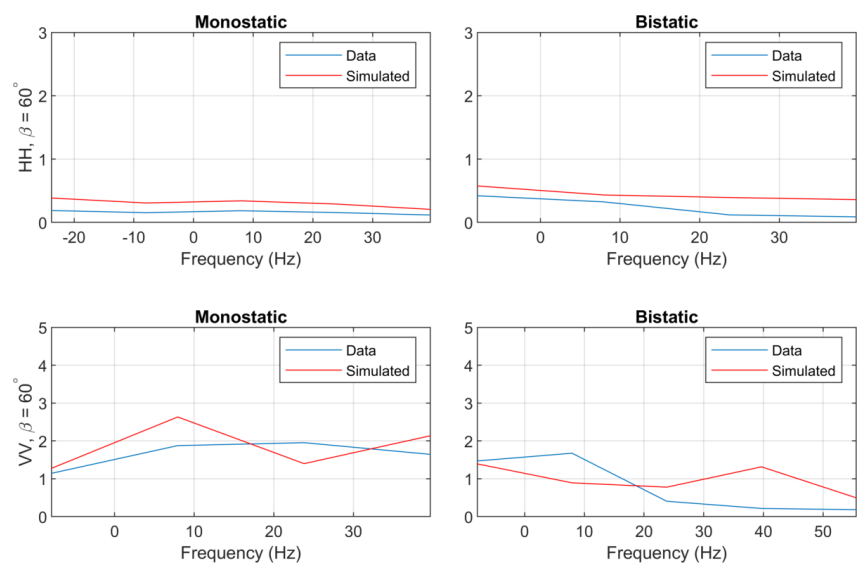

Fig. 4 - K-distribution shape parameter variation with frequency, $\beta=60^{\circ}$. Monostatic data on the left, bistatic on the right.

\section{DETECTION RESUlts}

In order to evaluate the performance of the single-channel NAMF, the multistatic M-NAMF and the M-maxrule detectors, a total of 8100 CPIs of $L=16$ pulses have been processed, where the model parameters have been estimated for each CPI and a new realization of the data generated.

The Probability of False Alarm $\left(P_{\mathrm{FA}}\right)$ after adaptive processing was first evaluated for the M-NAMF and M-maxrule detectors using simultaneous bistatic and monostatic data, and for the single-channel NAMF applied to the monostatic and bistatic data. Two values of the normalized Doppler frequency, $f_{d}$, are used: $f_{d}=0$ where the clutter is strong and $f_{d}=0.1$ (equivalent to $100 \mathrm{~Hz}$ ) where the noise dominates. Note that we can only show results down to $P_{\mathrm{FA}}=10^{-3}$ due to the limited number of samples.

Fig. 5 shows the $P_{F A}$-versus-threshold curves for both the horizontally and vertically polarized data. The values of $P_{\mathrm{FA}}$ are higher for $f_{\mathrm{d}}=0$ as the sea-clutter is stronger at the zeroDoppler bin (see Figs. 2-3). For nearly all the results, the $P_{\mathrm{FA}}$ values obtained from simulated data have a reasonable match with the real data except for the M-NAMF detector with $\beta=$ $60^{\circ}$ and VV polarization. For this case, the strong clutter result $\left(f_{d}=0\right)$ has a slight mismatch for the lower $P_{\mathrm{FA}}$ values (Fig. 5d).

The detection performance was analyzed by calculating the Probability of Detection $\left(P_{\mathrm{D}}\right)$ when a synthetic target was injected into both the real and simulated clutter data. The target has a Swerling-I fluctuation with a complex Gaussian amplitude and is completely correlated within a CPI. Note that the target is placed at the same range bin and Doppler frequency for each radar node in order to characterize the relative detection performance. The range cell under test is at the center of the intersection between the antenna patterns of the transmitter and bistatic receivers, which corresponds to $1900 \mathrm{~m}$ for $\beta=60^{\circ}$ and $1400 \mathrm{~m}$ for $\beta=60^{\circ}$.

The Receiver Operating Characteristics (ROC) in Fig. 6 show $P_{\mathrm{D}}$ as a function of $P_{\mathrm{FA}}$ for a signal-to-interference power ratio (SIR) of $10 \mathrm{~dB}$. When comparing the real and simulated results from each detector, the trends match quite closely. The best result is achieved for the data collected at $\beta$ $=60^{\circ}$ (Fig. 6a, $\mathrm{d}$ and Fig.6b, e), whereas the data at $\beta=90^{\circ}$ shows some mismatch (Fig 6.c, f). In general, the MNAMF detector shows better detection performance, followed by the M-maxrule. Both the multistatic detectors show improved performance over the single-channel NAMF when applied separately to the bistatic and monostatic data. 


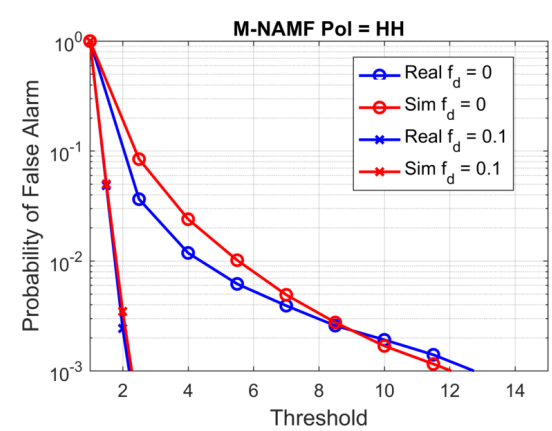

(a)

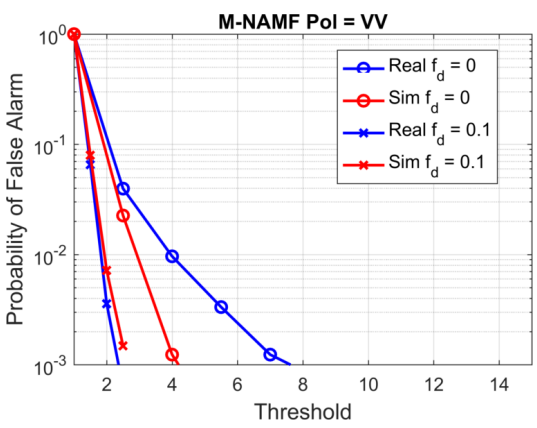

(d)

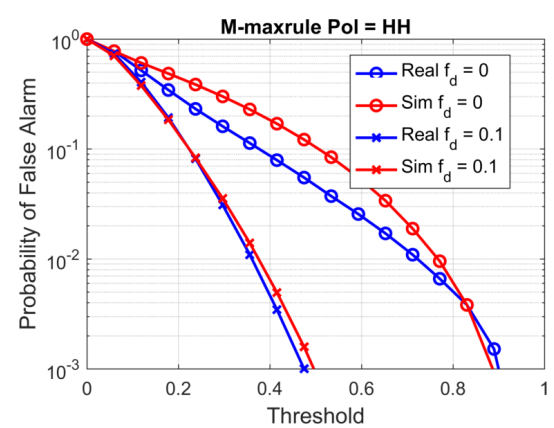

(b)

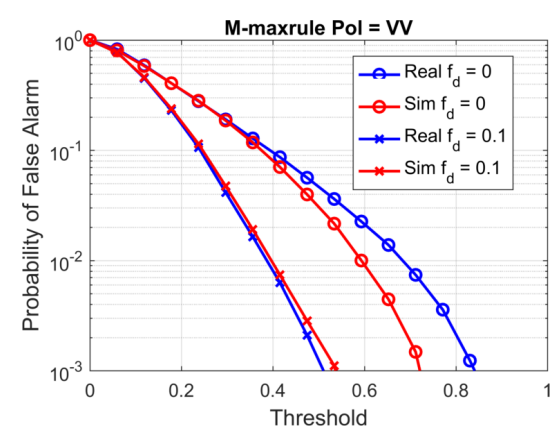

(e)

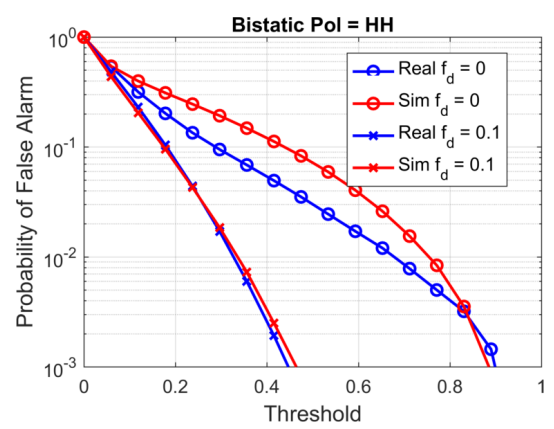

(c)

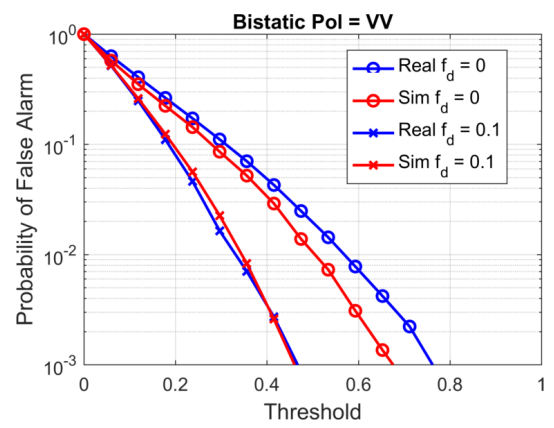

(f)

Fig. 5 - Probability of False Alarm for $\beta=60^{\circ}$ as a function of the detection threshold for real and simulated data and two normalized Doppler frequencies $\left(f_{\mathrm{d}}=0\right.$ and $\left.f_{\mathrm{d}}=0.1\right)$. Results show the multistatic NAMF detector with (a) HH and (d) VV data, Multistatic maxrule detector with (b) $\mathrm{HH}$ and (e) VV data and the single-channel NAMF detector with (c) HH and (f) VV bistatic data.

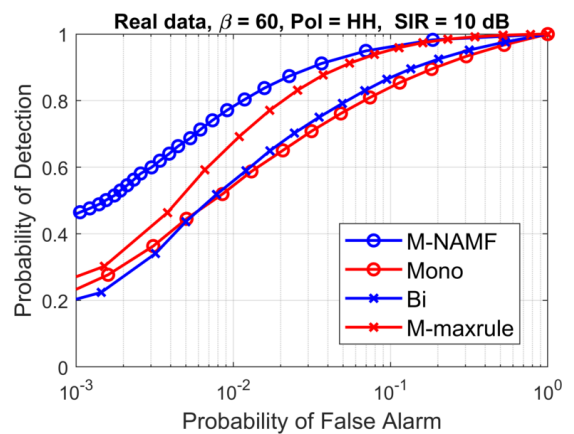

(a)

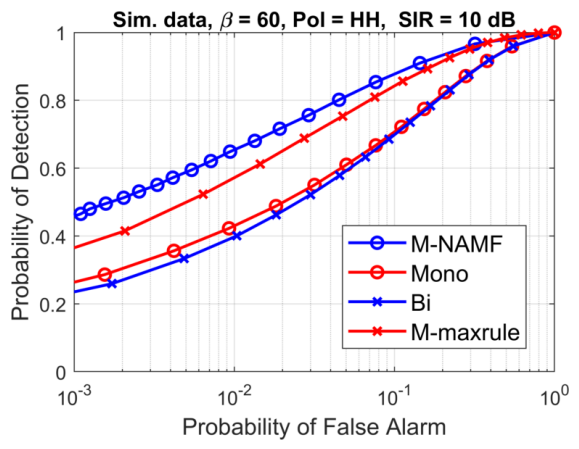

(d)

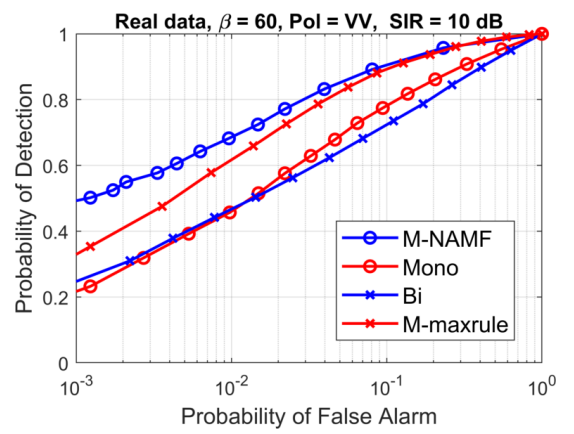

(b)

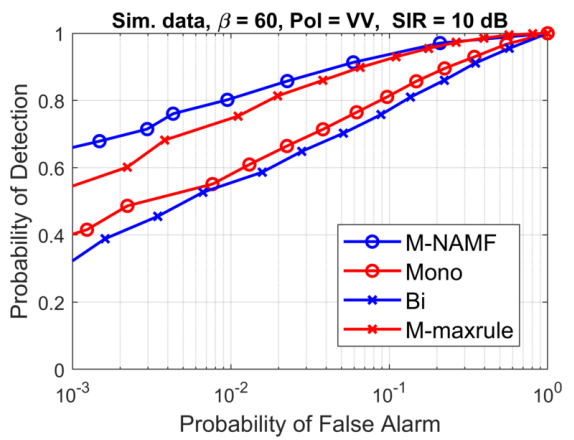

(e)

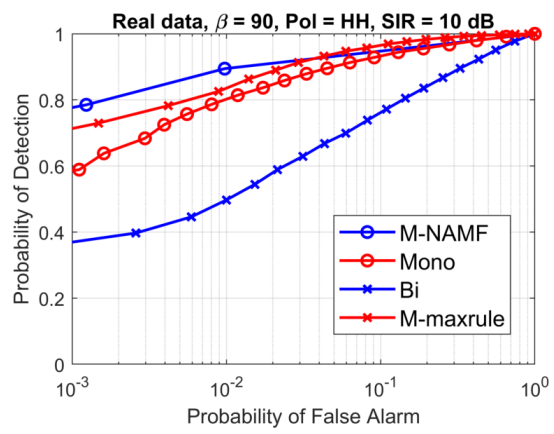

(c)

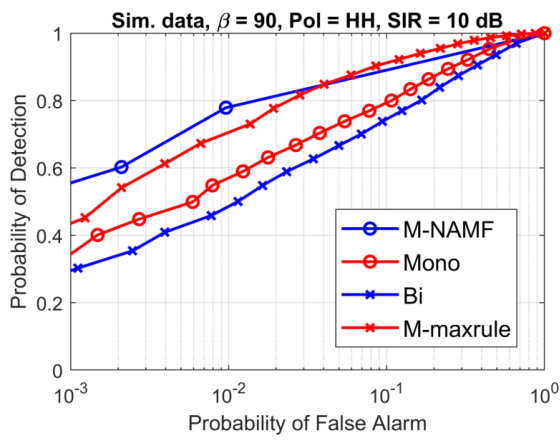

(f)

Fig. 6 - ROC curves with SIR $=10 \mathrm{~dB}$ and $f_{d}=0$ for the Multistatic NAMF and Multistatic maxrule detectors and the single-channel NAMF detector applied to monostatic and bistatic data. Results for the $\mathrm{HH}$ polarization at $\beta=60^{\circ}$ are shown for (a) real and (b) simulated data, VV polarization at $\beta=60^{\circ}$ for (b) real and (e) simulated data and $\mathrm{HH}$ polarization at $\beta=90^{\circ}$ for (c) real and (f) simulated data. 
The final result in Fig. 7 shows the probability of detection as a function of the SIR for a $\mathrm{P}_{\mathrm{FA}}$ of $10^{-3}$. To compare the relative detection performance, the required SIR at a fixed $P_{D}$ of 0.8 has been used. For the M-NAMF detector, the required SIR is $15 \mathrm{~dB}$ and $13 \mathrm{~dB}$ for the real and simulated data, respectively. For the other algorithms, the differences are all between 1-4 dB. The gain obtained with the M-NAMF technique is about $7 \mathrm{~dB}$ over the monostatic single-channel NAMF for both real and simulated data. This gain decreases to about $3.5 \mathrm{~dB}$ for the M-maxrule detector.

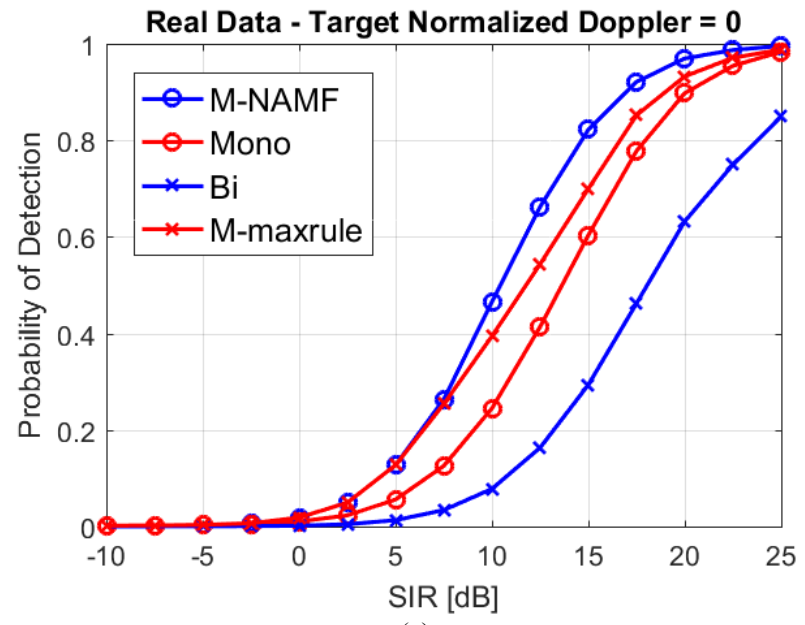

(a)

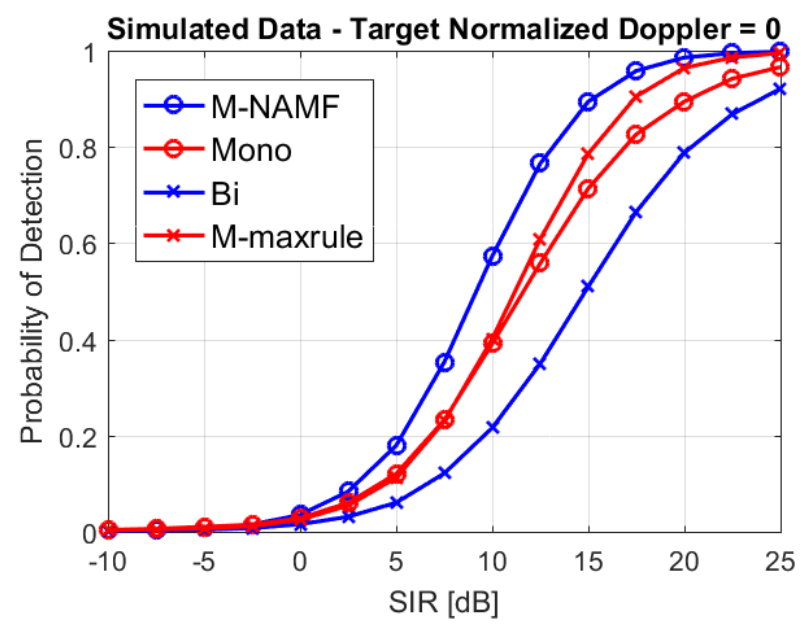

(b)

Fig. 7 - Probability of Detection as a function of SIR, for a fixed $\mathrm{P}_{\mathrm{FA}}=10^{-3}$. HH polarization, $\beta=60^{\circ}, f_{d}=0$, (a) real and (b) simulated data.

\section{CONCLUSION}

The first part of this paper has addressed the modelling of multistatic sea-clutter data, with a particular focus on their Doppler properties. The evolved Doppler spectrum model was used to generate synthetic data, which - along with the real data - were employed to evaluate the performance of two multistatic adaptive radar detectors. The accuracy of the simulation method has been demonstrated with an analysis of the $\mathrm{P}_{\mathrm{FA}}$ against detection threshold, the $\mathrm{P}_{\mathrm{D}}$ vs. $\mathrm{P}_{\mathrm{FA}}$ at a fixed SIR and the variation of the SIR at a fixed $P_{D}$. This comparison also demonstrated the relative performance of the multistatic detectors with the M-NAMF showing the best results, followed by the M-maxrule. Future work will focus on further improvements in the model fitting by utilizing a bimodal clutter spectrum.

\section{ACKNOWLEDGMENTS}

The authors would like to thank Dr Waddah Al-Ashwal, Dr Stephan Sandenbergh, Dr Matthew Ritchie, Prof Michael Inggs, Dr William Miceli and the rest of the team involved in the measurement campaign from the University of Cape Town and University College London.

\section{REFERENCES}

[1] Griffiths, H.D. and Palamà, R., 'Clutter Diversity', Chapter 6 in Novel Radar Techniques and Applications, Vol.2, (Klemm, R., Gierull, C., Griffiths, H.D., Lombardo, P. Koch, W. and Nickel, U. (eds)),IET, Stevenage, October 2017

[2] C. Y. Chong, F. Pascal, J.-P. Ovarlez, and M. Lesturgie, "MIMO Radar Detection in Non-Gaussian and Heterogeneous Clutter," IEEE J. Sel. Top. Signal Process., vol. 4, no. 1, pp. 115-126, 2010.

[3] R. Palamà, M. Greco, and F. Gini, "Multistatic adaptive CFAR detection in non-Gaussian clutter", EURASIP Journal on Advances in Signal Processing, pp.1-17, 2016.

[4] L. Rosenberg and Y. Abramovich, "Multi-static target detection in Kdistributed sea clutter and Gaussian noise" IEEE Radar Conference, Seattle, WA, 2017, pp. 0346-0351.

[5] F. Hoffmann, M. Ritchie, F. Fioranelli, A. Charlish and H. Griffiths, "Micro-Doppler based detection and tracking of UAVs with multistatic radar," 2016 IEEE Radar Conference (RadarConf), Philadelphia, PA, 2016, pp. 1-6.

[6] F. Fioranelli, M. Ritchie, S. Z. Gürbüz and H. Griffiths, "Feature Diversity for Optimized Human Micro-Doppler Classification Using Multistatic Radar," IEEE Transactions on Aerospace and Electronic Systems, vol. 53, no. 2, pp. 640-654, April 2017.

[7] M. Ritchie, A. G. Stove, S. Watts, K. Woodbridge, and H. D. Griffiths, "Application of a new sea clutter Doppler model," International Radar Conference, 2013, pp. $560-565$.

[8] M. Ritchie, A. Stove, A. Woodbridge, and H. Griffiths, "NetRAD: Monostatic and bistatic sea clutter texture and Doppler spectra characterization at S-band," IEEE Transactions on Geoscience and Remote Sensing, vol. 54, no. 9, pp. 5533-5543, September 2016.

[9] S. Watts, L. Rosenberg, S. Bocquet, and M. Ritchie, "The Doppler spectra of medium grazing angle sea clutter; part 1: Characterisation," IET Radar Sonar and Navigation, vol. 10, no. 1, pp. 24-31, 2016.

[10] F.Gini and M.Greco, "Covariance Matrix Estimation for CFAR detection in correlated heavy tailed clutter" Signal Processing, vol.82 no.12, 1847-1859, Dec 2002

[11] R. Palamà, M. S. Greco, P. Stinco and F. Gini, "Statistical analysis of bistatic and monostatic sea clutter" IEEE Transactions on Aerospace and Electronic Systems, vol. 51, no. 4, pp. 3036-3054, Oct. 2015.

[12] M. Ritchie, A. Stove, K. Woodbridge and H. Griffiths, "NetRAD: Monostatic and Bistatic Sea Clutter Texture and Doppler Spectra Characterization at S-Band" IEEE Transactions on Geoscience and Remote Sensing, vol. 54, no. 9, pp. 5533-5543, Sept. 2016.

[13] S. Watts, "Modeling and simulation of coherent sea clutter," IEEE Transactions on Aerospace and Electronic Systems, vol. 48, no. 4, pp. 3303-3317, October 2012.

[14] Bocquet, S., 'Parameter estimatoin for Pareto and K distributed clutter and noise', IET Radar Sonar and Navigation, vol. 9, no. 1, pp. $104-$ $113,2015$. 